\title{
Paediatric soft tissue sarcomas in a resource constrain setting: Grade and stage at presentation and at oncologic intervention are usually of poor prognostic characteristics.
}

\author{
Gabriel Ogun ${ }^{1}$, Uchenna O Ezenkwa ${ }^{1}$, Taiwo O Babatunde ${ }^{1}$, Michael C Nweke ${ }^{1}$, Omolade \\ O Adegoke ${ }^{1}$, Oludare O Olulana ${ }^{1}$, and Biobele J Brown ${ }^{1}$ \\ ${ }^{1}$ University of Ibadan College of Medicine
}

June 1, 2020

\begin{abstract}
Aim: To describe the pattern of paediatric soft tissue sarcomas with emphasis on the grade and stage at the first surgical or medical oncologic intervention. Methods: We retrospectively reviewed all cases of histologically confirmed paediatric Soft Tissue Sarcomas in the Department of Pathology, University College Hospital (UCH), Ibadan, Nigeria in children age 0-14 years. The study period was January 1991 to December 2016. Information obtained included age, gender, morphology and site of the tumours. The tumour grade and pathologic/clinical staging of all cases that had surgical biopsy or surgical resection were also obtained and verified from the clinical records. Tumour grading was carried out using the Fédération Nationale des Centres de Lutte Contre le Cancer (FNCLCC) Sarcoma group grading system and staging was done using TNM. Results: One hundred and twelve cases were seen with an almost equal male-to-female ratio and age range from 5 months to 14 years (median 8.5 years). The peak age group was 5-9 years. Rhabdomyosarcoma was by far the most common histological type (73.2\%). Other types were undifferentiated sarcomas (6.3\%), fibrosarcoma (5.4\%), Kaposi sarcoma (4.5\%), and $2.7 \%$ each for synovial sarcoma, dermatofibrosarcoma protuberans. The common primary sites were the head and neck (including the orbit) $51(45.5 \%)$, and the abdominopelvic $27(24.1 \%)$ regions. Other sites included the lower limb 13(11.6\%), trunk 12(10.7\%), upper limb 6(5.4\%). Majority (67.9\%) had histologic grade 3 at presentation based on the FNCLCC grading system. About $48.2 \%$ had Stage T2b while 9.8\% were stage T2a based on American Joint Committee on Cancer Staging (AJCC) system. Conclusion: Rhabdomyosarcoma, undifferentiated sarcomas and Kaposi sarcoma are the commonest paediatric malignant soft tissue tumours in our practice. Majority of our patients present with late stage tumour and with high histologic grade thereby conferring poor prognosis at presentation/first surgical or medical oncologic intervention.
\end{abstract}

\section{What's already known about this topic?}

Delayed presentation is usually associated with poor prognosis and stage of presentation of Soft tissue Sarcomas.

\section{What does this article add?}

Majority of patients in our practice setting presents with late stage tumour and with high histologic grade thereby conferring poor prognosis at presentation/first surgical or medical oncologic intervention.

\section{INTRODUCTION}

Soft tissue sarcomas (STS) are a heterogeneous group of tumours with diverse origin and histological subtypes. ${ }^{1}$ They are relatively rare tumours and are even rarer in paediatric patients. ${ }^{2}$ The crude incidence rate for Soft Tissue Sarcoma in children in Ibadan, Nigeria where most of our patients derive from is 0.56 per $100,000 .{ }^{3}$ Our practice setting is in a lower middle income environment, and as such general 
treatment of malignancies can be quite challenging. This is pertinent given the aggressiveness, tendency for recurrence and extensive battery of diagnostic methods needed for exact histologic diagnosis. The cost of health care is borne wholly by the patients without the support of any health insurance scheme. This is burdensome to the patient as well as the health system considering all the treatment modalities involved in managing soft tissue sarcomas. As a result, the best attempt practically at a good outcome in children with Soft Tissue Sarcoma is usually often at the first surgical excision. This is as a consequence of most patients defaulting subsequent adjuvant radiation and chemotherapy resulting in the patients being lost to follow up. ${ }^{4}$

The management and prognosis of Soft Tissue Sarcomas in children generally, is dependent on a wide surgical excision with histologically free tumour margins, as well as the pre-requisite grade and size of the respective tumours at presentation. ${ }^{1,5-7}$ This study aims to review the factors during the first surgical excision in our institution (namely tumour grade and size), which affect the long-term prognosis in paediatric patients histologically diagnosed with Soft Tissue Sarcoma.

\section{METHODS}

\section{Study group-}

We retrospectively reviewed all cases of histologically confirmed paediatric Soft Tissue Sarcomas in the Department of Pathology, University College Hospital (UCH), Ibadan, Nigeria. Our study population ranged in age from 0-14 years. The study spanned twenty six years from January 1991 to December 2016. The tumour grading and pathologic staging were done on all cases that had surgical biopsy or surgical resection. Cases that were not histologically confirmed as soft tissue sarcomas were excluded from the study.

\section{Histologic Evaluation (Diagnosis and Grading)}

Tumour diagnosis was based on the World Health Organization classification of soft tissue tumours. ${ }^{8}$ The tumour grade was assessed using the French Federation of Cancer Centers (Fédération nationale des centres de lutte contre le cancer; FNCLCC) system. ${ }^{9}$

\section{Tumour Staging}

The tumour staging was based on the 2010 American Joint Committee on Cancer (AJCC) staging, which incorporates the following: tumor size (T1[?]5 cm, T2 $>5 \mathrm{~cm}$ ) and depth (a-superficial, b-deep), nodal involvement $(\mathrm{N})$, distant metastases $(\mathrm{M})$, and histologic grade $(\mathrm{G}) .{ }^{10}$

\section{Statistical analysis}

The data generated was analyzed using descriptive statistics and presented as frequencies and mean.

\section{Ethical considerations}

This study was approved by the joint Institutional review board of the University of Ibadan and University College Hospital, Ibadan, Nigeria. Also, this study was performed in compliance with the guidelines of the Helsinki declaration on biomedical research on human subjects. Confidentiality of the identity of the patients and personal health information was maintained.

\section{RESULTS}

\section{Demographic Data}

One hundred and twelve cases of paediatric soft tissue sarcomas were seen within the study period comprising $58(51.8 \%)$ males and $54(48.2 \%)$ females. The male-to-female ratio was 1.1:1. Table 1 shows key summary of findings from the study.

The median age of the study population was 8.5 years with a range of 5 months to 14 years of age. Figure 1 shows the age groups of the population with 5-9 years being the modal age group.

\section{Histologic Tumour types.}


Table 2 shows the histologic types of the tumours seen. The commonest histologic tumour type was Rhabdomyosarcoma accounting for $73.2 \%$ of all tumours while giant cell tumour of soft tissue and alveolar soft part tumour were the least encountered ( $0.9 \%$ each). Among the 82 cases of rhabdomyosarcomas, the embryonal variant was predominantly seen $67(81.7 \%)$. Other variants documented were the alveolar $8(9.8 \%)$, and pleomorphic $4(4.9 \%)$ variants, $3(3.7 \%)$ cases were classified as rhabdomyosarcoma not otherwise specified (NOS). The vasoformative sarcomas comprised 5 cases of Kaposi sarcoma and 1 case each of angiosarcoma and haemangiopericytoma. Other types are as classified in Table 2. There was no remarkable gender difference in the occurrence of the tumour subtypes (Table 2). Males had 4 tumours more than the females.

\section{Tumour location}

As shown in Table 2, the commonest tumour location was the head and neck followed by the abdominopelvic region. Of the 51 head and neck tumours, the orbit was the commonest location with 23 (45.1\%). Twenty-four (88.9\%) out of the 27 tumours found in the abdominopelvic region were pelvic in location while the remaining $3(11.1 \%)$ occurred in the abdomen. Rhabdomyosarcoma occurred more in the head and neck (including the orbit) while the NRSTS occurred more in the extremities. Of the 51 tumours in the head and neck, 34 were rhabdomyosarcomas and these were predominantly embryonal rhabdomyosarcomas. Twenty out of the 27 abdominopelvic tumours were rhabdomyosarcomas, 18 of these were embryonal rhadomyosarcoma and were pelvic in location.

\section{Tumour Size.}

The tumour size ranged from $1-17 \mathrm{~cm}$ with a mean of $7.04+-4.62 \mathrm{~cm}$. The largest tumour was a dermatofibrosarcoma protuberans (DFSP).

\section{Tumour Grade and Stage}

Tumour grading was available for 104 tumours. Most of the tumours were of high grade. T2b stage was the commonest tumour stage followed by T1b stage and over half of the tumours were located in unfavorable sites (Table 1). No lymph node involvement or distant metastasis was observed.

\section{DISCUSSION}

Soft tissue sarcomas (STS) in children are rare tumours, approximately 1000 cases are diagnosed annually in the United States of America. ${ }^{11,12}$ These tumours are heterogenous in nature and can occur in any part of the body. ${ }^{13}$ This study documented 112 paediatric soft tissue sarcomas over a 26 year period, representing roughly 4.3 cases every year. This result is similar to an earlier reported figure of 4.7 per year from our centre. This study however differs with Dauda et al. (Jos Nigeria) and Kachanovet al. (Moscow) who reported 8.9 and 6.8 per year respectively in children less than 15 years of age ${ }^{14-16}$ This difference can be attributed to different study intervals. The studies by the afore mentioned authors (Dauda et al. and Kachanovet al. ) each covered a 10-year period compared to this present study. Another reason for this difference may be due to different disease demographics among different study populations.

Soft tissue sarcomas (STS) are classified broadly into Rhabdomyosarcomas( RMS) and nonrhabdomyosarcomas (NRMS) otherwise termed non-rhabdomyosarcoma soft tissue sarcomas (NRSTS). ${ }^{1,11}$ Most studies report rhabdomyosarcoma (RMS) as the commonest paediatric STS similar to this study $^{2,11,15-17}$ The predominance of embryonal and alveolar RMS supports the childhood occurrence of these sarcoma subtypes while the very few pleomorphic RMS supports the rare occurrence of these tumours in children. ${ }^{12,13}$ Other adult-type STS seen in this study differ somewhat in spectrum from those seen elsewhere. ${ }^{18}$ Leiomyosarcoma and liposarcoma reported by Ferrari et al . were not seen in this study. ${ }^{18}$ Also, Ferrari et al. reported synovial sarcoma as the commonest NRSTS tumours, accounting for $34 \%$ of their cases contrary to vascular sarcomas seen in this study as the commonest NRSTS. ${ }^{18}$ Other researchers have documented other sarcoma subtypes as being the commonest among the NRSTS supporting the heterogeneity of these tumours. ${ }^{1}$ Occurrence of these NRSTS in the paediatric age suggests similar yet to-be identified etiologic factors. 
No difference in tumour occurrence was seen between male and female gender in this study suggesting a common pathogenetic pathway. Studies have suggested genetic alterations such as Li Fraumeni syndrome and exposures to radiation as possible causes of RMS while the causes and risk factors for NRSTS are not known. ${ }^{11}$ The modal age in our study differs from those of Dauda et al. in Nigeria and Kachanov et al. in Moscow which documented older age groups. ${ }^{15,16}$ This difference is due to the differences in distribution of tumour subtypes across the age groups in these studies. In the study by Kachanov et al . RMS constituted $23 \%$ of tumours in the age group 10-14 years while the NRSTS tumours were predominant in this age group although RMS accounted for 54.4\% of all the STS. ${ }^{16}$ Likewise, alveolar RMS and Embryonal RMS occurred with higher frequency in the age group 12-15 years in the study by Dauda et al, compared to the 5-9 years seen in this present study. ${ }^{15}$

Anatomic location for STS varies in different studies. We documented head and neck as the commonest location. This is in contrast to Daudaet al . whose study reported extremities as the commonest location. ${ }^{15}$ This may suggest different pathogenesis. For example, embryonic cell arrest during migration in caudalcephalad direction with malignant transformation may explain the higher frequency of embryonal and alveolar rhabdomyosarcoma in such regions.

The known prognostic factors for STS we studied include tumour size, grade and stage, histological subtype and location. Ferrari et al . found among NRSTS, increasing tumour depth and size to be more strongly predictive of survival among children compared to their adult counterparts. ${ }^{18}$ Deep tumours and tumour size greater than $5 \mathrm{~cm}$ were associated with poorer survival while tumours of the head and neck showed better survival. ${ }^{18}$ Tumour size not only determines tumour burden but also surgical resection as a modality of treatment and this factor affects tumour recurrence. ${ }^{12}$ Rhabdomyosarcomas are by their nature high grade tumours although very chemo- and radiosensitive. ${ }^{13}$ Overall, most STS in this study were of high grade status. The mean tumour size of $7.4 \mathrm{~cm}$, higher frequency of grade 3 tumours and tumour stage suggests worse prognosis for tumours seen in our practice environment. On the other hand, the higher frequency of head and neck location of STS might suggest a favourable prognosis in addition to the predictability of response to therapy if offered to these patients. ${ }^{12}$ Patients with deep tumours or tumour size $>5 \mathrm{~cm}$ may therefore need to be treated with multimodal approach. ${ }^{19}$

Absence of follow up data in this study has made it difficult to examine the effects the poorer clinicopathological parameters with patient overall survival. Prospective studies should incorporate data on adjuvant therapies and survival as these may suggest additional biological behaviours of tumours in our environment.

\section{Conclusion}

Rhabdomyosarcoma, undifferentiated sarcomas and Kaposi sarcoma are the commonest paediatric malignant soft tissue tumours in our centre. Majority of our patients present with late stage tumour and with high histologic grade thereby conferring poor prognosis at presentation/first surgical or medical oncologic intervention.

\section{Acknowledgement: None}

\section{Funding: None}

\section{References}

1. Spunt SL, Skapek SX, Coffin CM. Pediatric nonrhabdomyosarcoma soft tissue sarcomas. Oncologist. 2008;13(6):668-78.

2. Ferrari A, Sultan I, Huang TT, Rodriguez C, Casanova M, Spunt SL. Soft Tissue Sarcoma Across the Age Spectrum: A Population- Based Study from the Surveillance Epidemiology and End Results Database. Pediatr Blood Cancer. 2011;57(6):943-9.

3. Thomas JO, Aghadiuno PU. Nigeria: Ibadan Cancer Registry, 1985-1992. In: Parkin DM, Krama'rova' E, Draper DG, et al., eds. International Incidence of Childhood Cancer, volume II. Lyon: IARC Publications, no 144. 1998. 43-5 p. 
4. Anyanwu SNC, Egwuonwu OA, Ihekwoaba EC. Acceptance and adherence to treatment among breast cancer patients in Eastern Nigeria. Breast. 2011;20:S51-3.

5. Ferrari A, Casanova M, Collini P, Meazza C, Luksch R, Massimino M, et al. Adult-type soft tissue sarcomas in pediatric-age patients: experience at the Istituto Nazionale Tumori in Milan. J Clin Oncol. 2005;23(18):4021-30.

6. Spunt SL, Poquette CA, Hurt YS, Cain AM, Rao BN, Merchant TE, et al. Prognostic factors for children and adolescents with surgically resected nonrhabdomyosarcoma soft tissue sarcoma: an analysis of 121 patients treated at St Jude Children's Research Hospital. J Clin Oncol. 1999;17(12):3697-705.

7. Khoury JD, Coffin CM, Spunt SL, Anderson JR, Meyer WH, Parham DM. Grading of nonrhabdomyosarcoma soft tissue sarcoma in children and adolescents. Cancer. 2010;116(9):2266-74.

8. Vilanova JC, Girona U De. WHO Classification of Soft Tissue Tumors. 2017. 187-195 p.

9. Guillou BL, Coindre J, Bonichon F, Bui NB, Terrier P, Collin F, et al. Comparative Study of the National Cancer Institute and French Federation of Cancer Centers Sarcoma Group Grading Systems in a Population of 410 Adult Patients With Soft Tissue Sarcoma. J Clin Oncol. 1997;15(1):350-62.

10. Edge SB, Compton CC. The American Joint Committee on Cancer : the 7th Edition of the AJCC Cancer Staging Manual and the Future of TNM. Ann Surg Oncol. 2010;17:1471-4.

11. Loeb DM, Thornton K, Shokek O. Pediatric Soft Tissue Sarcomas. Surg Clin North Am. 2014;88(3):118.

12. Agarwala S. Pediatric rhabdomyosarcomas and nonrhabdomyosarcoma soft tissue sarcoma. J Indian Assoc Pediatr Surg. 2006;11(1):15-23.

13. Winette P, Graaf TA Van Der, Orbach D. Soft tissue sarcomas in adolescents and young adults : a comparison with their paediatric and adult counterparts. Lancet Oncol. 2017;18(3):166-75.

14. Babatunde TO, Akang EEU, Ogun GO, Brown BJ. Pattern of childhood cancer in University College Hospital , Ibadan during 1991 - 2010 and comparison with the previous three decades. Paediatr Int Child Health. 2015;35(2):144-50.

15. Dauda M, Yakubu D, Mandong B. Sarcomas in Nigerian Children in Jos North Central Nigeria. Afr J Med Med Sci. 2015;43(Suppl 1):37-44.

16. Kachanov DY, Dobrenkov K V, Abdullaev RT, Shamanskaya T V, Varfolomeeva SR. Incidence and Survival of Pediatric Soft Tissue Sarcomas in Moscow Region, Russian Federation , 2000 - 2009. Sarcoma 2012;2012:350806. doi: 10.1155/2012/350806..

17. Aliyu UM, Okuofo EC, Okwonna CO, Sahabi SM. Clinicopathological pattern of soft tissue sarcoma in a tertiary health institution in North Western Nigeria. Int J Res Med Sci. 2018;6(5):1632-8.

18. Ferrari A, Miceli R, Casanova M, Gronchi A, Collini P, Meazza C, et al. Adult-type soft tissue sarcomas in paediatric age : A nomogram-based prognostic comparison with adult sarcoma. Eur J Cancer. 2007;3(43):2691-7.

19. Casali PG, J.-Y. B. clinical practice guidelines Soft tissue sarcomas : ESMO Clinical Practice Guidelines for diagnosis , treatment and follow-up clinical practice guidelines. Ann Oncol. 2010;21(Supplement 5):198203.

Table 1: Profile of cases of soft tissue sarcoma with respect to gender, location, histologic subtypes, grade and stage

Variable

Gender $(n=112)$ Male Female 
Variable

Location(n=109) Abdominopelvic Trunk Head and neck (including orbit) Lower limb Upper limb Not documented

Histologic subtype $(\mathbf{n}=\mathbf{1 1 2})$ Rhabdomyosarcoma Vasoformative sarcoma Synovial sarcoma MPNST DFSP Fibrosarcom Tumour grade Grade 1 Grade 2 Grade 3 Unknown

pT Stage T1a T1b T2a T2b Not documented

Anatomic site stage Favorable site Unfavorable Not documented

Table 2: Occurrence of the tumour subtypes according to gender.

\begin{tabular}{lll}
\hline Histologic subtype & Male & Female \\
\hline Rhabdomyosarcoma $(\mathrm{n}=82)$ & 41 & 41 \\
Vasoformative sarcoma $(\mathrm{n}=7)$ & 4 & 3 \\
Fibrosarcoma $(\mathrm{n}=6)$ & 4 & 2 \\
Synovial sarcoma $(\mathrm{n}=3)$ & 1 & 2 \\
MPNST $(\mathrm{n}=2)$ & 0 & 2 \\
DFSP $(\mathrm{n}=3)$ & 2 & 1 \\
Giant cell Tumour of soft tissue $(\mathrm{n}=1)$ & 0 & 1 \\
Alveolar soft part sarcoma $(\mathrm{n}=1)$ & 1 & 0 \\
Undifferentiated pleomorphic sarcoma $(\mathrm{n}=7)$ & 4 & 3 \\
\hline
\end{tabular}

\section{Figure Legend}

Figure 1: Chart showing the age distribution of the patients.

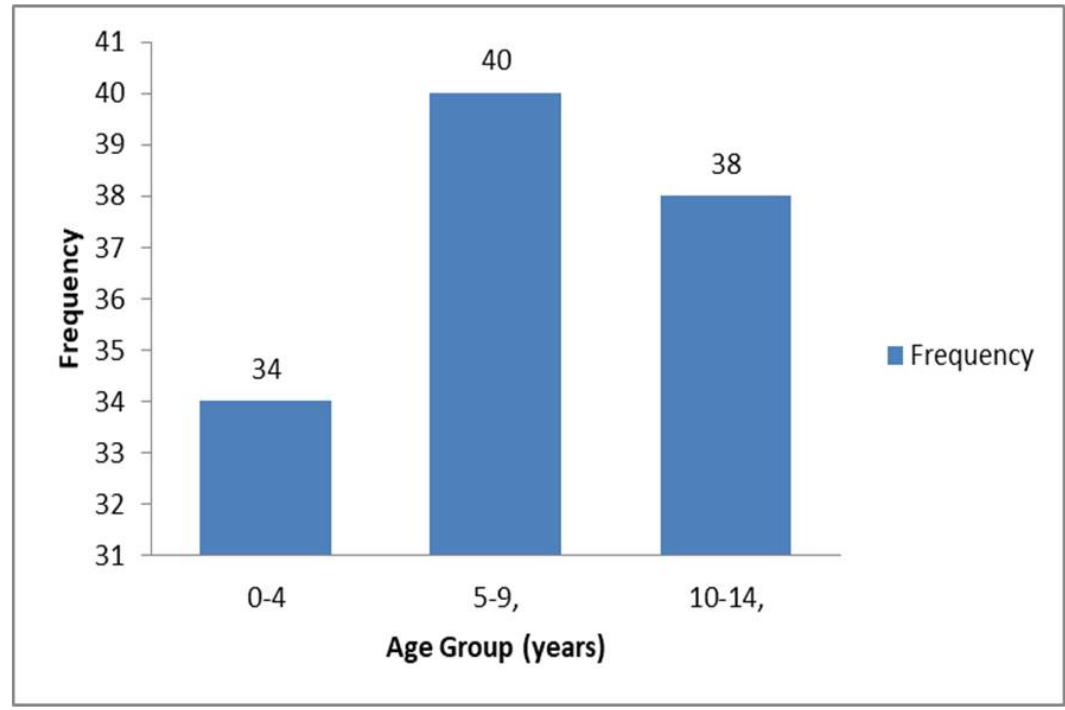

